\title{
Adiabatic Effectiveness and Heat Transfer Coefficient of Shaped Film Cooling Holes on a Scaled Guide Vane Pressure Side Model
}

\author{
Jan Dittmar, Achmed Schulz, and Sigmar Wittig \\ Institut für Thermische Strömungsmaschinen, Universität Karlsruhe (TH), Karlsruhe, Germany
}

The demand of improved thermal efficiency and high power output of modern gas turbine engines leads to extremely high turbine inlet temperature and pressure ratios. Sophisticated cooling schemes including film cooling are widely used to protect the vanes and blades of the first stages from failure and to achieve high component lifetimes. In film cooling applications, injection from discrete holes is commonly used to generate a coolant film on the blade's surface.

In the present experimental study, the film cooling performance in terms of the adiabatic film cooling effectiveness and the heat transfer coefficient of two different injection configurations are investigated. Measurements have been made using a single row of fanshaped holes and a double row of cylindrical holes in staggered arrangement. A scaled test model was designed in order to simulate a realistic distribution of Reynolds number and acceleration parameter along the pressure side surface of an actual turbine guide vane. An infrared thermography measurement system is used to determine highly resolved distribution of the models surface temperature. An in-situ calibration procedure is applied using single embedded thermocouples inside the measuring plate in order to acquire accurate local temperature data.

All holes are inclined $35^{\circ}$ with respect to the model's surface and are oriented in a streamwise direction with no compound angle applied. During the measurements, the influence of blowing ratio and mainstream turbulence level on the

Received 25 June 2002; accepted 1 July 2002.

This study was partly funded by the Ministry of Research and Technology of the Federal Republic of Germany through the joint research program 'AG-Turbo' and the MTU Aero Engines GmbH, Munich, Germany.

Address correspondence to Jan Dittmar, Institut für Thermische Strömungsmaschinen, Universität Karlsruhe (TH), Kaiserstrasse 12, Karlsruhe, 76128, Germany. E-mail: jan.dittmar@its.uni-karlsruhe.de adiabatic film cooling effectiveness and heat transfer coefficient is investigated for both of the injection configurations.

Keywords Gas turbine, Film Cooling, Adiabatic film cooling effectiveness, Heat transfer coefficient, Shaped film cooling holes

\section{INTRODUCTION}

In modern gas turbines, sophisticated cooling schemes including film cooling are widely used to protect the vanes and blades of the first turbine stages from failure and to achieve high life cycles. In film cooling applications, injection from discrete holes is commonly used to generate a coolant film on the blade's surface. In an attempt to improve the cooling process, recent attention has been given to contouring the injection hole geometry. Modern manufacturing technologies, such as precise electric-discharge machining or laser drilling, enable one to form the injection hole into more complex shapes.

Many of the earlier studies of film cooling considered injection from a single row of discrete holes. Due to the threedimensional character of the flow field downstream of the coolant injection, the cooling effectiveness decreases compared to injection from a continuous slot. In order to improve the lateral distribution of the injected coolant and to approach a two-dimensional film cooling situation, more studies were focused on the injection from a double row of cylindrical holes (Jabbari et al., 1978; Jubran and Brown, 1985; Jubran and Maiteh, 1999). In general, the results show that for the same injected mass flow rate per unit span, the double row arrangement provides better cooling effectiveness compared to injection from a single row. Staggered rows show better performance than rows with inline arrangement. The gain in effectiveness is attributed to the lower penetration of the coolant jets because of lower momentum ratio resulting from the increased injection area and better lateral spreading of the cooling air. Increasing the distance between the two rows gives a significant decrease to both local and lateral averaged effectiveness, especially close behind the downstream row. A compound 
angle orientation of the holes, especially that of the second row, increases cooling effectiveness (Ligrani et al., 1994). Recent studies on film cooling holes with a diffuser-shaped expansion at the exit portion of the hole ("fanshaped" holes) have shown a promising improvement of the film cooling performance. Various research groups (Goldstein et al., 1974; Gritsch et al., 1997, 1998; Makki and Jakubowski, 1986; Yu et al., 1999; Reiss and Bölcs, 1999) investigated film cooling effectiveness with injection from different hole shapes, including holes with a lateral or forward expanded exit part. They all found higher effectiveness values for the shaped holes compared to cylindrical holes. The lateral expanded holes show much better lateral spreading of the injected coolant and hence more uniform distribution of effectiveness. Due to the reduced jet exit momentum, shaped holes show less penetration of the coolant jet into the mainstream and reduced velocity gradients in the mixing region (Thole et al., 1996).

The present study aims to compare a single row cooling configuration, including shaped holes, with a double row of cylindrical holes in a realistic flow field, typical for the pressure side surface of a turbine guide vane. A similar study has been conducted for a suction side model and results for the adibatic film cooling effectiveness have been presented in Dittmar et al. (2000).

\section{EXPERIMENTAL SET-UP}

The experiments presented in this article are conducted on a model of a pressure side of an actual turbine guide vane assembled in an open loop atmospheric wind tunnel (see Figure 1) Its contoured shape has been designed in order to simulate almost realistic distribution of the Reynolds number

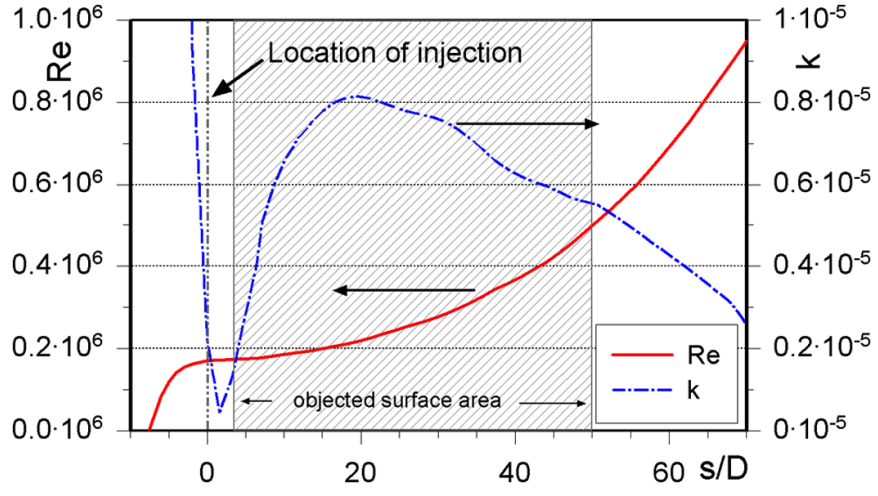

FIGURE 2

Distribution of Reynolds number and acceleration parameter k.

and acceleration parameter $\mathrm{k}$ on the model's surface, as shown in Figure 2. The Reynolds number based on the chord length at the location of injection is about $1.8 \times 105$ and about 2400 based on the injection hole diameter. The model essentially consists of three parts: (1) a base block, (2) an interchangeable injection module, and (3) a contoured test plate for surface temperature measurements. The test plate is made of a high temperature plastic (TECAPEEK ${ }^{\circledR}$ ) with a low thermal conductivity of about $0.43 \mathrm{~W} / \mathrm{m} \cdot \mathrm{K}$. In the top wall, three sapphire windows are inserted to enable optical access to the test model. Figure 3 shows a photo of the contoured test model assembled inside the test section.

In this study, the cooling performance of a single row of 8 fanshaped holes was compared to the performance of a double row of standard cylindrical holes in staggered arrangement

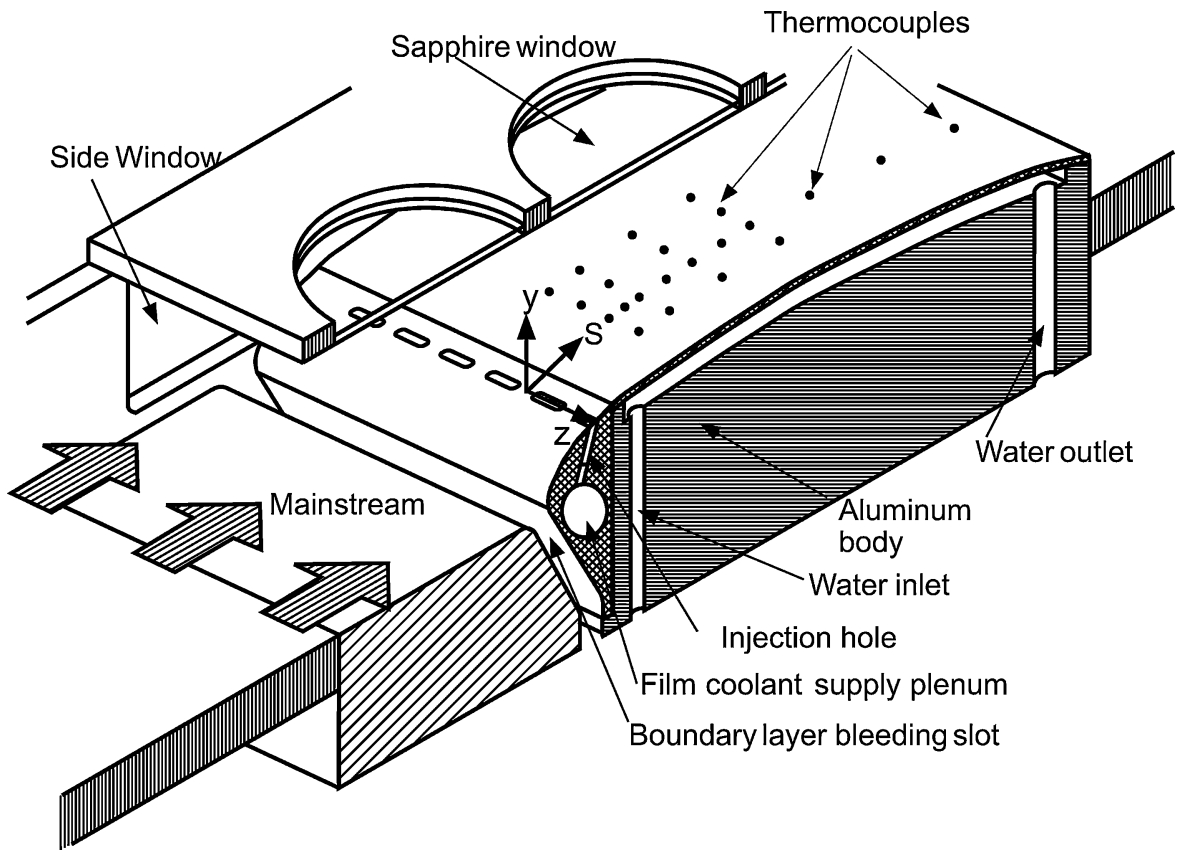

FIGURE 1

Experimental setup and large scale film cooling model. 


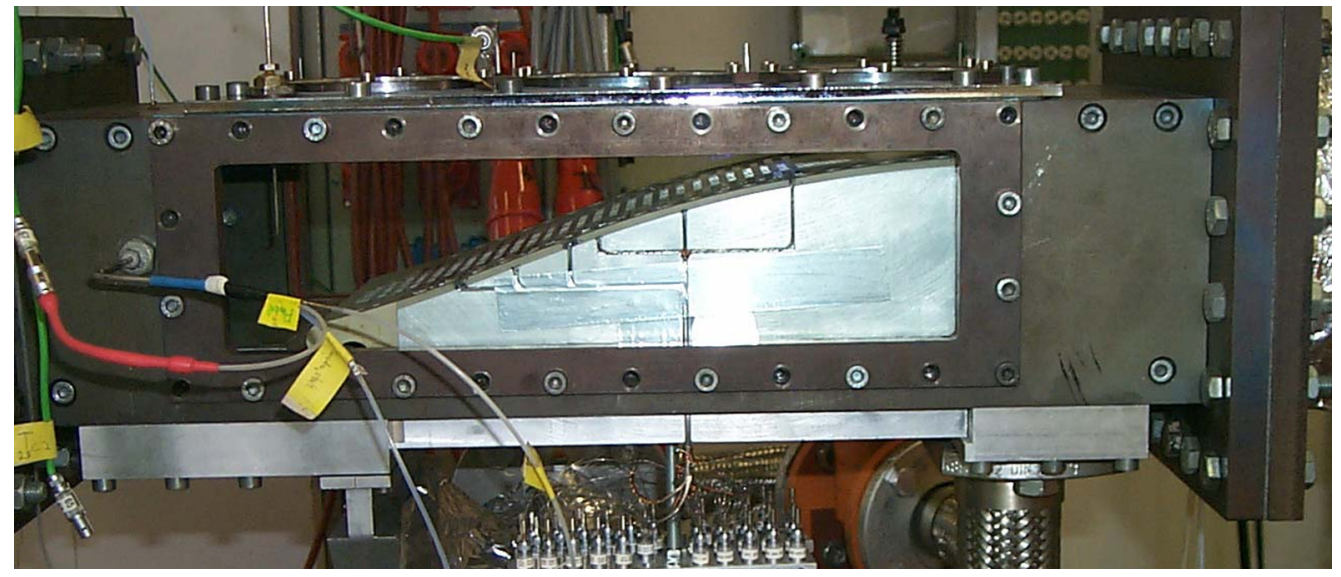

FIGURE 3

Photo of assembled pressure side film cooling test section.

including 16 holes in total. Details of the hole geometry are shown in Figure 4. The holes are placed at $10 \%$ of the chord length from the stagnation point of the blade model. Both hole types have a diameter of $4 \mathrm{~mm}$ at the inlet part of the hole and are inclined $35^{\circ}$ in the streamwise direction. The total length of the the holes is 6 diameters. The fanshaped holes feature a cylindrical hole inlet with a length of 2 diameters. Following, the hole is laterally expanded with an opening angle of $14^{\circ}$ on each side. In each row the holes are separated 4 diameters in the lateral direction.

\section{MEASUREMENT TECHNIQUE}

Surface temperature measurements are performed by means of an infrared thermography system (AGEMA ${ }^{\circledR}$ Thermovision $900 \mathrm{SW}$ ). The IR-system consists of an optical scanner which

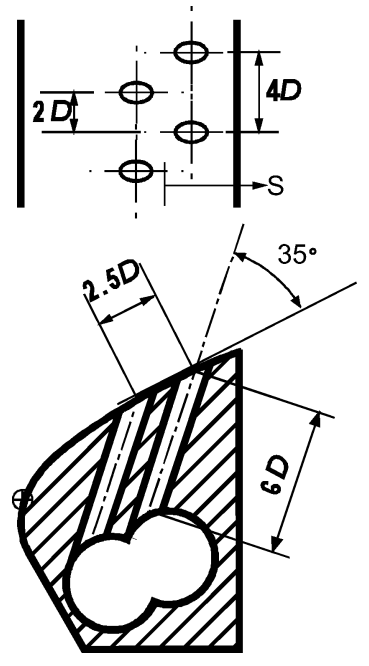

(a)

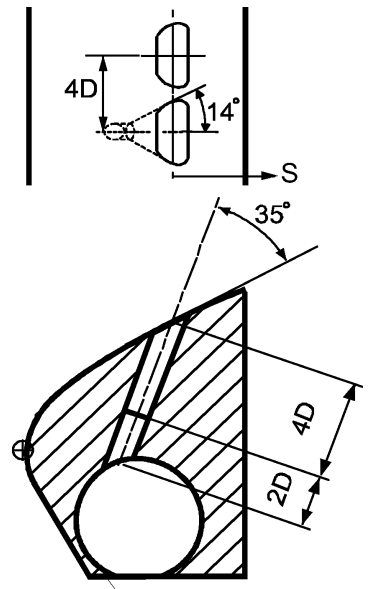

(b)
FIGURE 4

Investigated film cooling injection geometry. directs the incoming infrared radiation line by line on the detector working in a wavelength bandwidth of $2-5.4 \mu \mathrm{m}$. The scanner is mounted above the test section of the wind tunnel and reads the surface temperature information through the sapphire windows. The output signal of the IR-detector is digitized by a processing computer in a frame of $136 \times 272$ pixels corresponding to a spatial resolution of $0.65 \times 0.65 \mathrm{~mm}$ with the optical setup used in the experiments. The surface of the contoured test plate is covered with a black paint with a well-known emissivity of $\varepsilon=0.95$.

In order to get quantitatively accurate wall temperature data, the radiation data from the IR-system is recalibrated using 12 thermocouples embedded flush with the surface. A calibration routine has been developed taking reflected ambient radiation as well as transmission losses into account. The total amount of infrared radiation detected by the IR-system, $I_{\text {tot, }}$ can be summarized as follows (see Figure 5):

$$
I_{\text {tot }}=\tau \cdot \varepsilon \cdot I_{w}+\tau \cdot(1-\varepsilon) \cdot I_{\text {sur }}
$$

$I_{w}$ is the emitted infrared energy from the test plate and $I_{\text {sur }}$ is the consolidated radiation from all the surrounding that is reflected from the test plate (reflectivity $=(1-\varepsilon)$ ). The parameter $\tau$ is the overall transmission factor (surface to detector) and $\varepsilon$ is the emissivity of the test plate (covered with black paint). It is assumed that no infrared radiation is transmitted through the test plate and any infrared radiation from the hot mainflow itself is neglected. To correlate wall temperature $T_{w}$ and the emitted infrared radiation $I_{w}$, a semi-empirical relation is used:

$$
I_{w}=\frac{R}{e^{\left(B / T_{w}\right)}-F}
$$

The factors $R, B$, and $F$ are calibration factors provided with the AGEMA ${ }^{\circledR}$ IR-system, taking the transmission behavior of different lenses and various measuring ranges of the detector into account. Combining Equations (1) and (2), the surface 


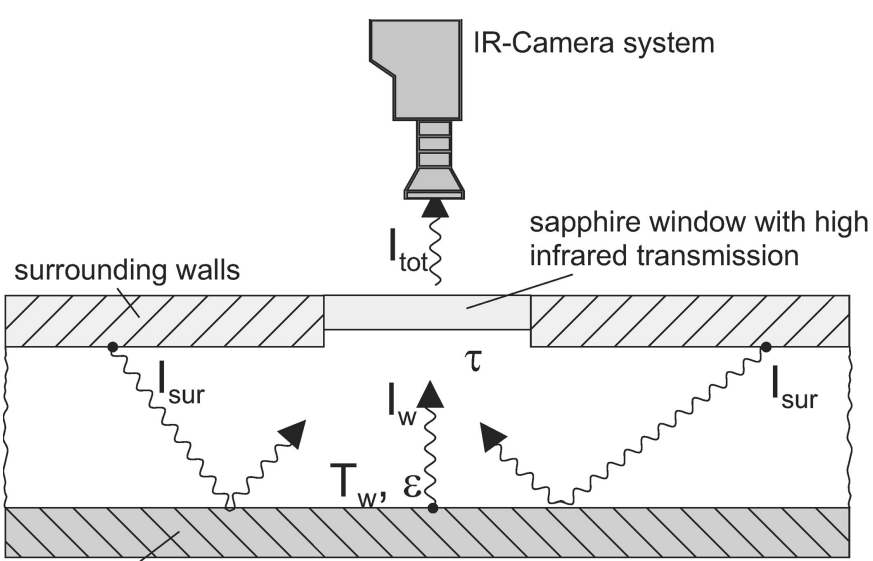

Test plate

FIGURE 5

Modeling of infrared radiation inside the test section.

temperature from the test plate can be calculated if the radiation of the surrounding $I_{\text {sur }}$ and the overall transmission factor $\tau$ are known:

$$
T_{w}=\frac{B}{\ln \left[\frac{R \cdot \tau \cdot \varepsilon}{I_{\mathrm{tot}}-\tau \cdot(1-\varepsilon) \cdot I_{\mathrm{sur}}}+F\right]}
$$

For accurate measurements a more sophisticated procedure is needed especially in situations were the parameters $\tau$ and $I_{\text {sur }}$ are difficult to determine because of their spatial variation. Therefore, additional temperature data obtained with embedded thermocouples is used to recalibrate the IR-data. For each thermocouple location, values for $\tau$ and $I_{\text {sur }}$ were derived by minimizing the difference of thermocouple and IR-temperature value. The averaged values for $\tau$ and $I_{\text {sur }}$ that give the best result in $n$ thermocouple locations are determined by using a least error squares method:

$$
\begin{gathered}
\Psi^{2}=\sum_{i=1}^{n}\left[T_{\mathrm{TC}, \mathrm{i}}-T_{w, i}\left(\begin{array}{c}
\tau \\
I_{\text {sur }}
\end{array}\right)\right]^{2} \equiv \min \\
\Rightarrow \frac{\partial \Psi^{2}}{\partial\left(\tau, I_{\text {sur }}\right)}=0
\end{gathered}
$$

To calculate the best fitting values for $\tau$ and $I_{\text {sur }}$, the system of $\mathrm{n}$ nonlinear equations is solved by using a robust numerical algorithm based on the Levenberg-Marquardt procedure. A detailed description of this mathematical procedure is given in Press et al. (1988).

An example result of the IR-calibration procedure for a typical case with coolant injection is shown in Figure 6. The wall temperatures of the test plate measured with the help of the IR system after calibration show very good agreement with those determined by the thermocouples. The temperature difference is less than $1 \%$ for the vast majority of measured test cases.

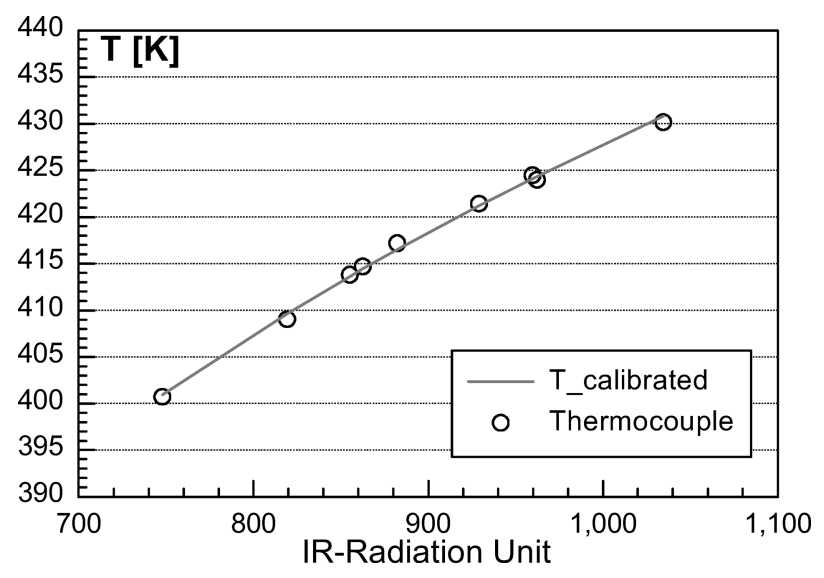

FIGURE 6

Typical result of an in-situ calibration of a IR-camera data set.

\section{DETERMINATION OF FILM COOLING PARAMETERS}

In contrast to usual two-temperature heat transfer problems, film cooling is dominated by three driving temperatures: the temperature of the hot gas, the temperature of the injected coolant gas, and the resulting wall temperature. The heat transfer situation to a film cooled surface is commonly described by

$$
\dot{q}_{w}=h_{f} \cdot\left(T_{\mathrm{aw}}-T_{w}\right)
$$

with the adiabatic wall temperature $T_{\mathrm{aw}}$ as a reference temperature. The adiabatic wall temperature is typically presented as the non dimensional adiabatic film cooling effectiveness (Goldstein, 1971):

$$
\eta=\frac{T_{m}-T_{\mathrm{aw}}}{T_{m}-T_{c}}
$$

Thus, the film cooling effectiveness describes the cooling potential of the injected film without any heat flux into the wall. The heat transfer coefficient $h_{f}$ in Equation 5 considers the influence of the coolant injection on the heat transfer process due to the modified fluid dynamics and is independent from the temperature boundary layer condition. Both of these two parameters have to be known to calculate the wall heat flux accurately.

In a real film cooling situation it is not possible to separate the two parameters $\eta$ and $h_{f}$. Alternatively, the heat transfer to the wall can be described as follows:

$$
\dot{q}_{w}=h(\theta) \cdot\left(T_{m}-T_{w}\right)
$$

where $T_{m}$ denotes the local mainstream temperature as a reference value. Here, all the effects of the cooling film are described with the value of the heat transfer coefficient $h(\theta)$ as a function of the non dimensional wall temperature $\theta$ (Choe et al., 1974):

$$
\theta=\frac{T_{m}-T_{c}}{T_{m}-T_{w}}
$$

Metzger et al. (1968) and Gritsch et al. (1999) proved that the relationship $h(\theta)$ is linear and valid even in realistic gas 


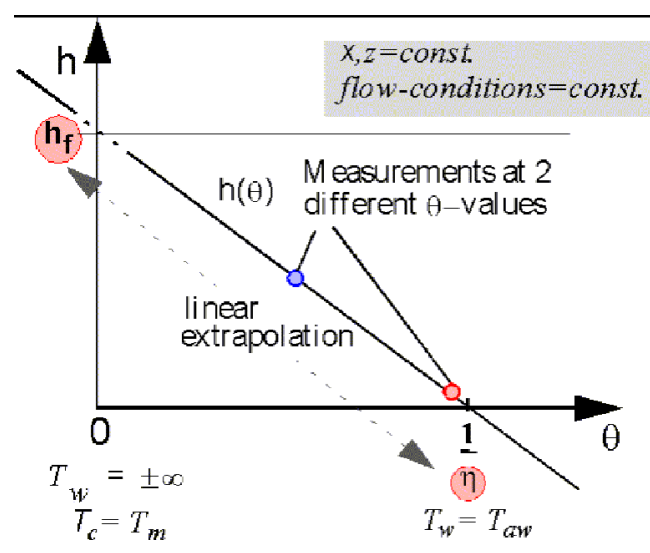

FIGURE 7

Using the linear superposition principle for the determination of $\eta$ and $h_{f}$.

turbine conditions. The linear function can be specified by the equation

$$
h(\theta)=h_{f} \cdot(1-\eta \cdot \theta)
$$

which is visualized in Figure 7. The point of intersection of the straight line with the abscissa represents the reciprocal value of the adiabatic film cooling effectiveness (adiabatic condition). The intersection with the ordinate represents the heat transfer coefficient $h_{f}$. Within the experiments, the linearity of Equation (9) is used to determine the two basic film cooling parameters $\eta$ and $h_{f}$. Therefore, two different sets of measurements of the surface temperature are performed. The first one is made at nearly adiabatic conditions without any additional cooling of the test plate. At identical flow conditions, a second measurement is made where the bottom side of the test plate is cooled with water in order to reduce the wall temperature $T_{w}$ and thereby the value of $\theta$. In both casess, the surface temperature data obtained with the IR-camera system is subsequently used to perform a threedimensional finite element heat flux analysis of the test plate. The calculated heat flux is then used to determine local values for $h(\theta)$ at each case. The radiative heat exchange inside the test section is taken into account to correct all the heat transfer data. Finally, the two $h(\theta)$-values are fitted with a straight line and the film cooling effectiveness $\eta$ as well as the heat transfer coefficient $h_{f}$ are extrapolated using the fitted line.

\section{RESULTS AND DISCUSSION}

Using the measurement technique and post processing procedure described above, film cooling effectiveness and heat transfer coefficient data is determined for both of the injection hole geometries. During the experiments, the blowing ratio $M$ was varied in the range of $0.2-1.5$ in the case of the double row with cylindrical holes and in the range of $0.25-3.0$ for the single row with fanshaped holes. The definition of the blowing ratio $M$, which is given in the nomenclature, is based on the inlet area of the injection holes as a reference area. Beside the blowing ratio variation, the influence of an increased mainstream turbulence level is investigated. The variation of the mainstream turbulence level of $T U=4 \%, 6 \%$, and $9 \%$ is achieved by the use of different grids in front of the test model. The temperature ratio of mainstream gas to coolant gas $T_{m} / T_{c}$ has been kept constant at a value of 1.3 during all the experiments by heating up the mainstream gas to about $440 \mathrm{~K}$ using electrical heaters.

\section{Adiabatic Film Cooling Effectiveness Results}

Figures 8 and 9 show local patterns of the adiabatic film cooling effectiveness at a mainstream turbulence intensity of
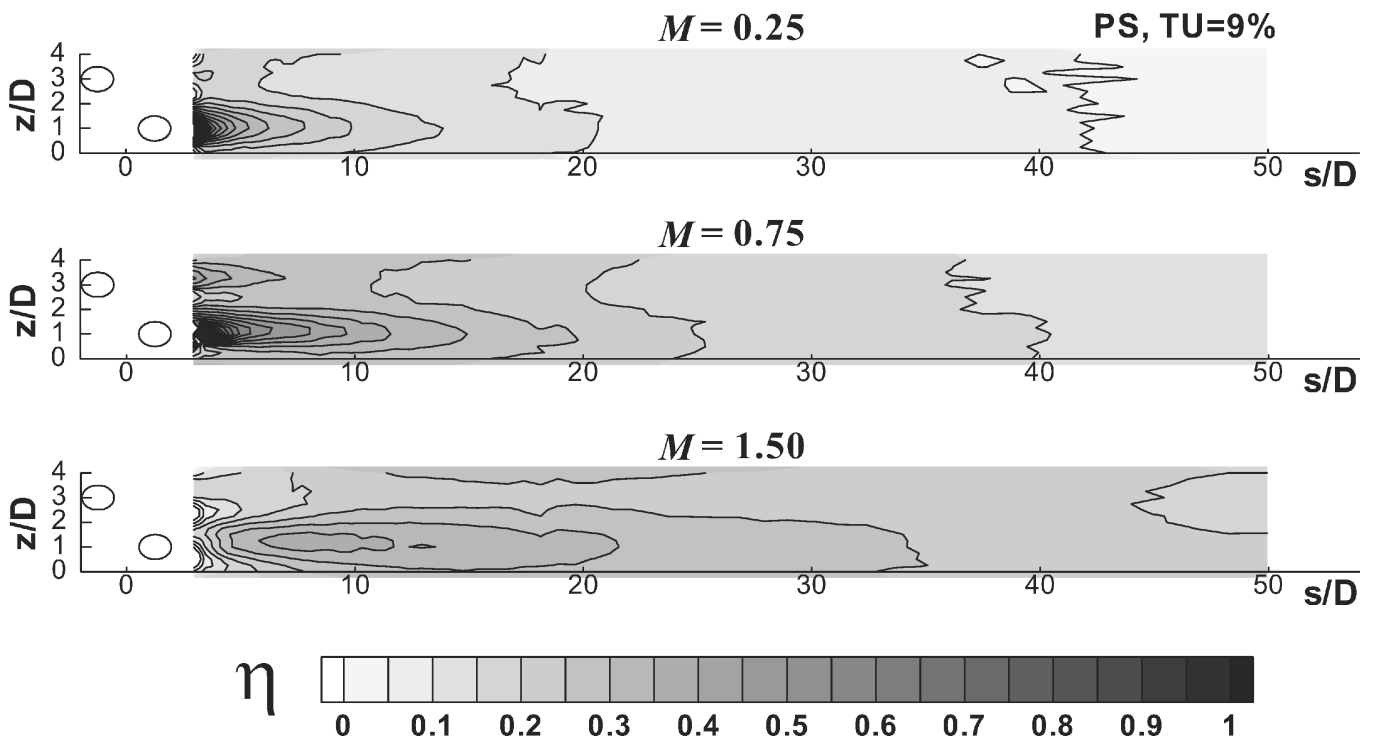

FIGURE 8

Local adiabatic effectiveness for the double row of cylindrical holes. 

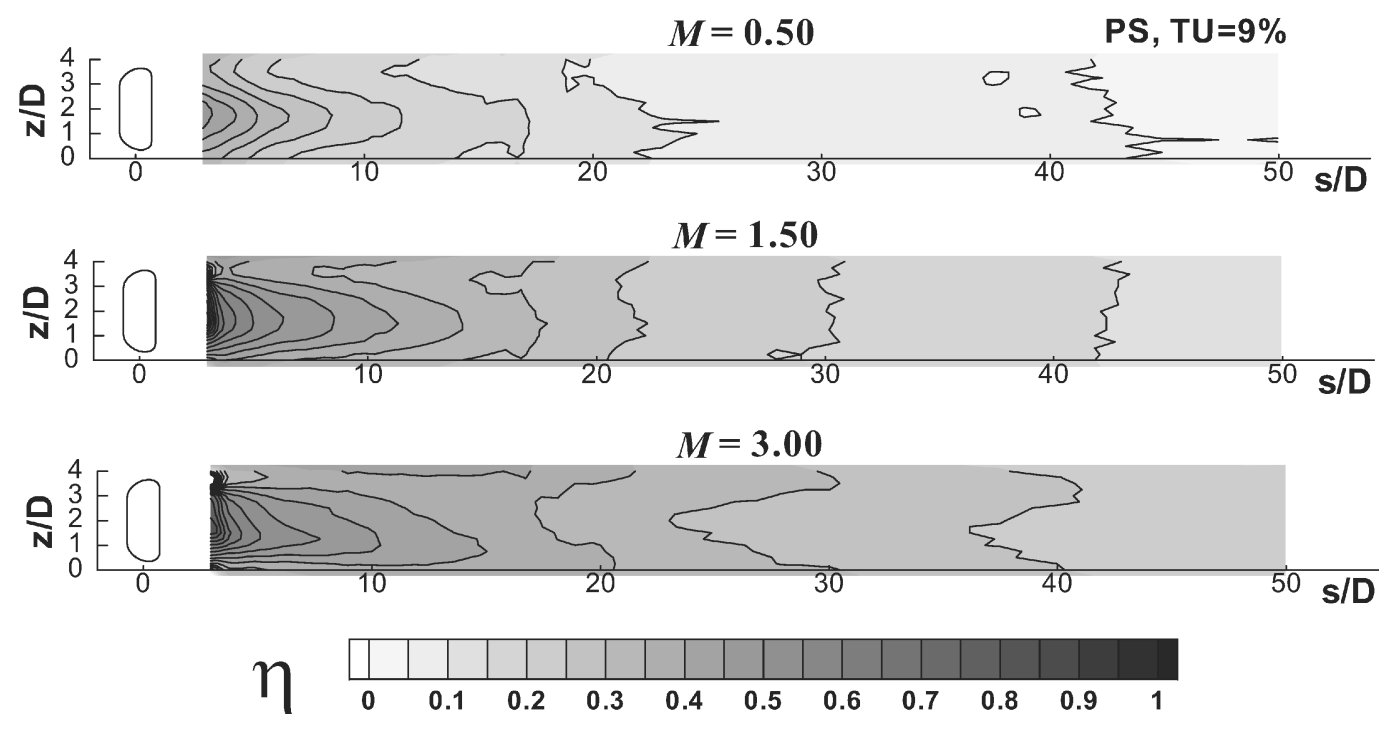

FIGURE 9

Local adiabatic effectiveness for the single row of fanshaped holes.

$9 \%$ downstream of the point of injection up to $s / D=50$ for the cylindrical holes and the shaped holes, respectively. At each case, results for a small, medium, and large blowing ratio value are plotted. For the single row with fanshaped holes, a blowing ratio combination of $M=0.5,1.5$, and 3.0 has been chosen. For the double row with cylindrical holes, a combination of $M=0.25,0.75$, and 1.5 has been chosen, which represents the same injected amount of total coolant mass flow per unit span.

In the case of the shaped holes, the results show very good effectiveness values especially close behind the injection. Due to the lateral expansion of the hole, the injected coolant is evenly spread which results in a quite uniform surface temperature distribution. An increasing blowing ratio causes increasing film cooling effectiveness for almost the total range of blowing ratio studied. At high blowing ratios of 3.0, the effectiveness decreases only slightly in an area very close downstream the injection holes. The double row of cylindrical holes shows a good performance in the low and medium blowing ratio range (up to $M=1.0$ ). Due to the staggered arrangement of the two rows, the coolant is well spread in the lateral direction and a quite uniform distribution of effectiveness is achieved. At higher blowing ratios $(M>1)$, the cooling jets start to separate from the wall which is more pronounced for the first row due to a blockage effect of the second row. A wake region right behind the injection is established and hot mainstream gas is transported to the wall caused by complex vortex generation, mainly in the shear layer between jet and mainstream. In this blowing ratio range, the adiabatic effectiveness is decreasing drastically in the near holes region as far as $x / D=15$.

Comparing the two different configurations for the same amount of coolant massflow injected indicates the superior cooling performance of the shaped holes. In the low blowing ratio range the cylindrical holes show likewise cooling effectiveness but at higher blowing ratios the shaped holes do have clear advantages due to the coolant jet separation of the cylindrical hole injection. The superior performance of the shaped holes at moderate and high blowing ratios, especially up to $s / D=30$, is emphasized looking at the lateral averaged effectiveness data (see Figure 10). The averaging is done over the range of one pitch including a distance of four hole diameters:

$$
\eta_{\text {Lat }}=\frac{1}{4} \cdot \int_{z / D=0}^{z / D=4} \eta(s / D, z / D) d z / D
$$

The improvement in adiabatic film cooling effectiveness when using shaped holes is caused mainly by the reduction of the jet exit momentum. The reduced penetration of the jet into the

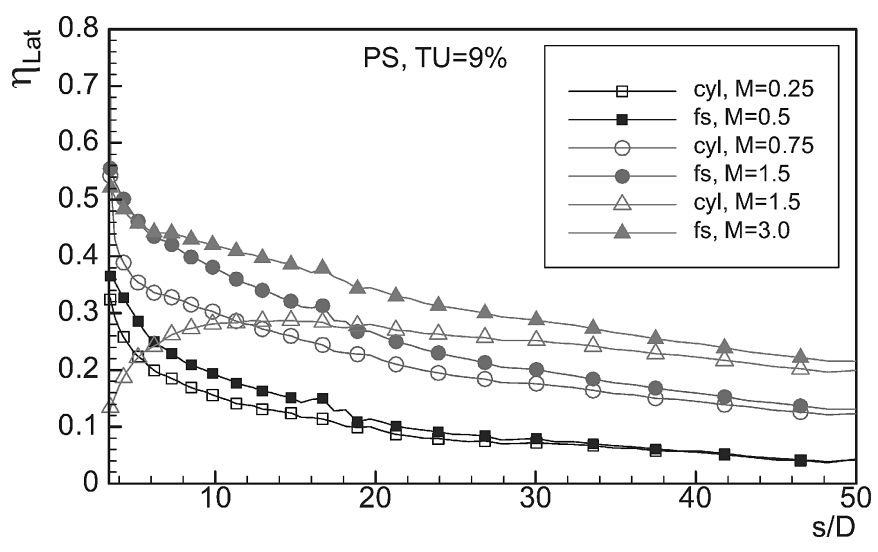

FIGURE 10

Lateral averaged adiabatic effectiveness for both of the hole configurations. 


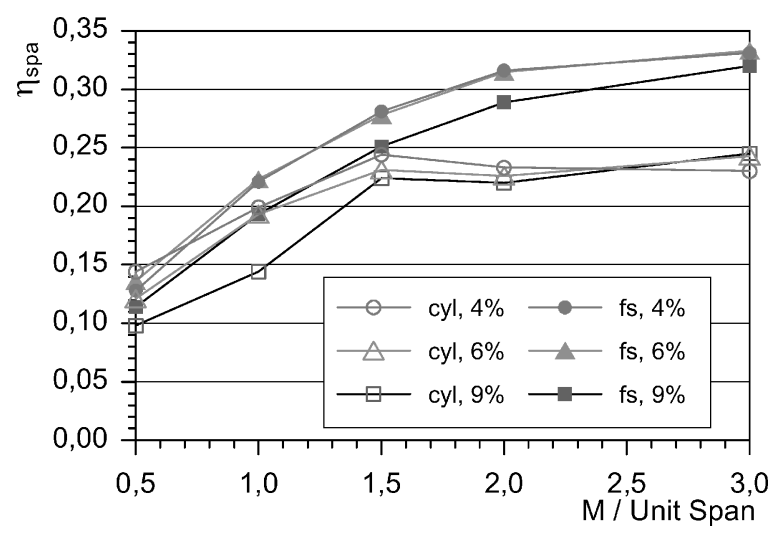

FIGURE 11

Space averaged adiabatic effectiveness for both of the hole configurations: Influence of mainstream turbulence level.

mainstream and the improved lateral spreading of the jet results in increased cooling effectiveness. Figure 11 shows space averaged data of film cooling effectiveness with varying blowing ratio at the various mainstream turbulence intensities of $T U=$ $4 \%, 6 \%$, and $9 \%$. The space averaged value is calculated by using the lateral averaged effectiveness data in a streamwise range up to $x / D=50$ :

$$
\eta_{\mathrm{spa}}=\frac{1}{50} \cdot \int_{s / D=0}^{s / D=50} \eta_{\mathrm{Lat}}(s / D) d(s / D)
$$

The data is plotted versus the blowing ratio per unit span in order to compare the two injection geometries at equivalent injected coolant mass flow. The space averaged data show the overall effectiveness that can be achieved on the cooled surface and allows a qualitative assessment of the two injection types. The influence of coolant jet separation for the cylindrical holes is clearly visible: The overall surface protection increases up to a optimal blowing ratio of about $M=0.75$. For higher blowing ratios, the space averaged effectiveness is nearly constant. Due to the coolant jet separation, the effectiveness decreases drastically in the near hole region. Further downstream, some of the injected coolant attaches to the surface again and leads to slightly increasing effectiveness. In total, this combination causes an almost constant space averaged effectiveness. In contrast to this, the overall surface protection is steadily increasing for the fanshaped holes and approaches its maximum effectiveness at the highest blowing ratio.

Enhanced mainstream turbulence intensity causes a drop in effectiveness for both cooling configurations at least up to moderate blowing ratios. The increased mainstream turbulence level enforces the mixing between coolant and mainstream and causes a faster decay in effectiveness in the streamwise direction. The increase of the turbulence level from 6 to $9 \%$ seems to have a much more detrimental effect than the increase from 4 to $6 \%$. In the case of the fanshaped holes, the influence of the higher turbulence level is decreasing with increasing blowing ratio. In the case of the cylindrical holes at high blowing ratios, the influence of turbulence level seems to be much weaker or even reverse. It is assumed that the lift-off phenomena is responsible for this behavior. In the case of jet separation, the enhanced mixing due to higher turbulence transports the injected cooling air back to the wall which reduces the wall temperature compared to high momentum injection into a less turbulent mainstream.

\section{Heat Transfer Coefficient Results}

Besides the effectiveness data, the heat transfer coefficient with coolant injection $h_{f}$ is of interest when discussing the total film cooling performance. The heat transfer coefficient data is determined by using the linear relationship in Equation (9) and is extrapolated from the two single measurements at different $\theta$-values. Figure 12 shows the distribution of lateral averaged heat transfer coefficients versus the streamwise distance $s / D$ at a mainstream turbulence intensity of $T U=9 \%$. The results are plotted for the two investigated configuration at three different blowing ratio values. Additionally, the heat transfer coefficient $h_{0}$, with no film injection but including the holes, is presented for both holes types. The results show significant peak values and high streamwise gradients at the beginning of the test plate. The high heat transfer coefficients are believed to be caused by a turbulent boundary layer state which is likely released by the presence of the holes. Due to the high acceleration in this region ( $k \cong 6 \cdot 10^{-6}$ at $s / D=7.5$ ), the boundary layer is forced back to the laminar state and the heat transfer coefficient is steadily decreasing until $s / D=30$. Both of the two hole configurations show rather the same distribution for the heat transfer coefficient $h_{0}$ without any coolant injection. Thus, the hole geometry shape in principle does not seem to have an important influence on the heat transfer. The results for the cases with coolant injection show all increased heat transfer compared to the case without injection. For the fanshaped holes, increasing the blowing ratio from the small to the medium value causes slight increase in the heat transfer coefficient $h_{f}$. A further increase of the blowing

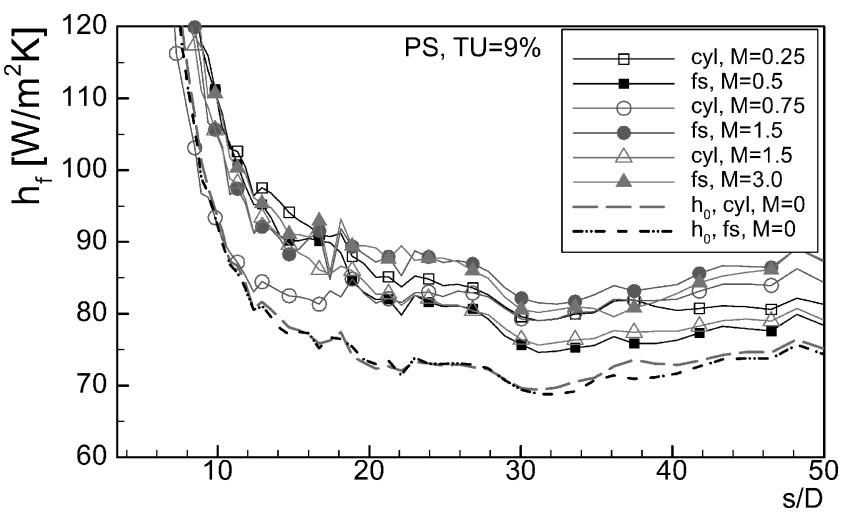

FIGURE 12

Lateral averaged heat transfer coefficient for both injection configurations. 

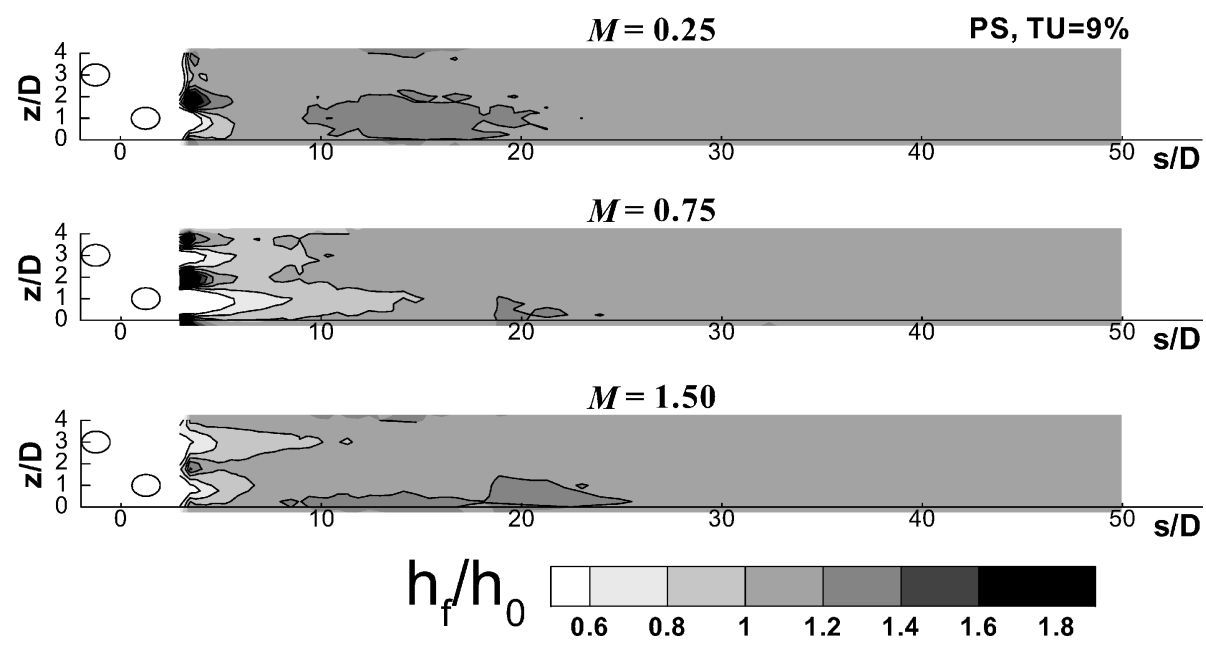

FIGURE 13

Non dimensional local heat transfer coefficient for the double row of cylindrical holes.

ratio to high values does not have a significant effect on the lateral averaged heat transfer coefficient. In the case of the double row of cylindrical holes, there is no clear influence of the blowing ratio. Increasing blowing from small to medium range causes a drop in the heat transfer coefficient in the near hole region but an increase in values of $h_{f}$ further downstream. The decrease in the far downstream region is reinforced at a high blowing ratio of $M=1.5$. One reason for this dependency on the blowing ratio in the case of the cylindrical holes might be the coolant jet lift-off at higher blowing ratios. As long as the cooling air stays attached to the surface, the influence on the heat transfer is more pronounced in the near hole region. As the jet detaches from the surface, the interaction of coolant gas and wall become less close behind the hole but become more pronounced in the downstream region. In general, one can state that the influence of the blowing ratio on the heat transfer coefficient $h_{f}$ is not as important as on the adiabatic film cooling effectiveness.

Figures 13 and 14 show local heat transfer data for the double row of cylindrical holes and the single row of fanshaped holes, respectively. Here, the heat transfer coefficient with film injection $h_{f}$ is normalized with the value without any injection $h_{0}$ in order to visualize the local influence on the heat transfer behavior. For the cylindrical holes, the heat transfer pattern shows a decrease of the heat transfer coefficient downstream of the centerline of the holes but an increase between the holes. This augmentation between the holes is mainly caused by 2 facts:

1. The film injection causes a blockage to the mainstream at the discrete hole positions. Therefore, the mainstream gas is diverted and accelerated between the holes which leads to
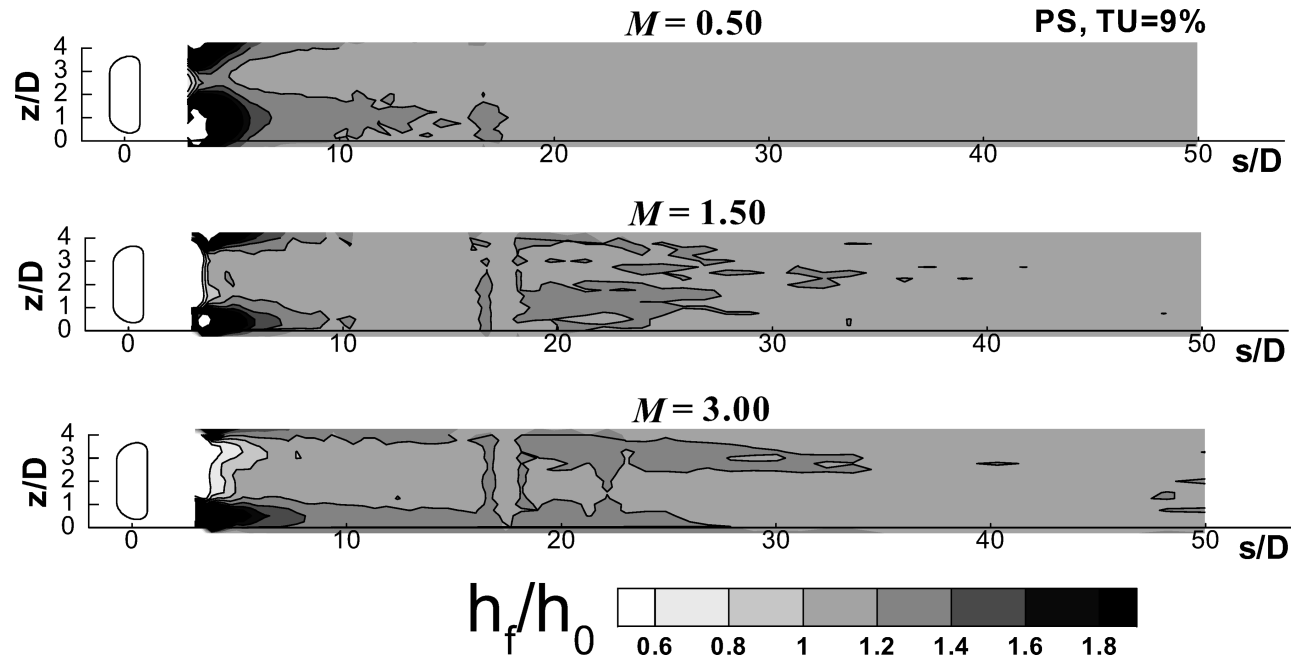

FIGURE 14

Non dimensional local heat transfer coefficient for the single row of fanshaped holes. 
higher local Reynolds numbers and thus higher heat transfer coefficients.

2. Additionally, the injection from discrete cylindrical holes is dominated by a counter-rotating vortex pair which is generated at the lateral edges of the coolant jet close behind the point of injection (Thole et al., 1996). The vortices of two adjacent holes leads to an increased turbulence level between the holes near the wall and thus to increased heat transfer coefficients. The development of the heat transfer coefficient indicates the dependence on the blowing ratio: At the medium blowing ratio of $M=0.75$ where a high film cooling effectiveness is achieved, the influence on the heat transfer is strongest. At a higher blowing ratio of $M=1.5$, the jet has already separated from the surface and thus causes less augmentation of the heat transfer coefficient. The results for the fanshaped holes show, in principle, the same behavior but with different influence of the blowing ratio. The main areas with increased heat transfer are also located at the lateral edges of the injected coolant jet. Even when the strength of the counter rotating vortex pair was found to be less for these type of hole (Thole et al., 1996), the mainstream blockage effect is stronger due to the higher surface coverage of the fanshaped holes. As there is less tendency of coolant jet lift-off found, the influence of injection on the heat transfer coefficient is steadily increasing with increasing blowing ratio, especially more downstream.

Figure 15 shows lateral averaged data for the non dimensional heat transfer enhancement due to cooling air injection. In general, the augmentation of the heat transfer coefficient $h_{f}$ is within $20 \%$ of its value without injection.

\section{Overall Film Cooling Performance}

In order to get an overall validation of film cooling, the adiabatic film cooling effectiveness and the heat transfer augmentation both have to be considered. Therefore, an overall film

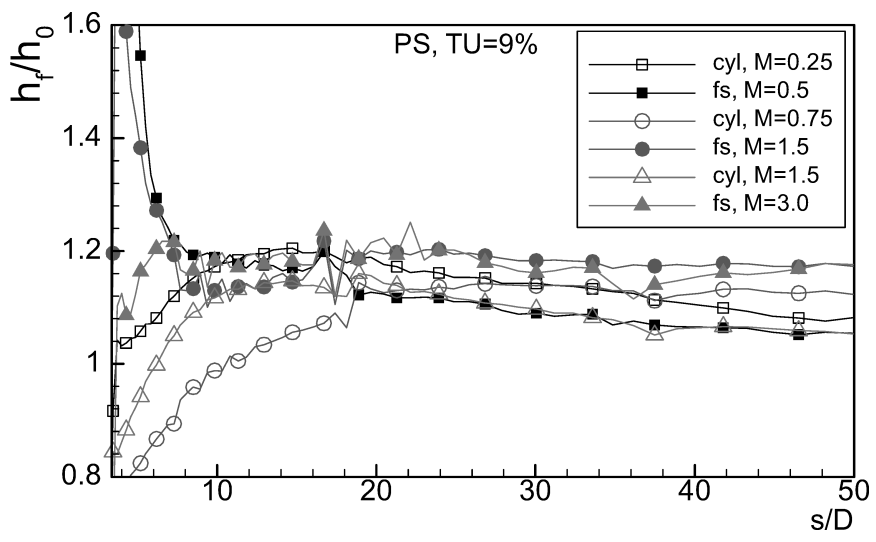

FIGURE 15

Non dimensional lateral averaged heat transfer coefficient for both injection configurations. cooling parameter, the Net Heat Flux Reduction parameter, is defined (Sen et al., 1996):

$$
\mathrm{NHFR}=\frac{\dot{q}_{0}-\dot{q}_{w}}{\dot{q}_{0}}=1-\frac{\dot{q}_{w}}{\dot{q}_{0}}
$$

Using the film cooling effectiveness $\eta$ and the heat transfer coefficient $h_{f}$ leads to:

$$
\mathrm{NHFR}=1-\frac{h_{f}}{h_{0}} \cdot(1-\eta \cdot \theta)
$$

As the NHFR parameter combines both major thermal effects, it is suitable to compare two different injection configurations by using only one parameter. Figure 16 shows the lateral averaged distribution of the NHFR parameter versus the non dimensional streamwise distance for the three different blowing ratio ranges. Here, $\theta$-value of 1.5 is used for the calculation of NHFR. It can be seen that for the low and medium blowing ratio range the overall cooling performance is similar for the two hole geometries and the same cooling air mass flow injected. Only a small benefit is achieved when using fanshaped holes. For the cylindrical holes at a blowing ratio of $M=0.25$, the injection even has a small negative effect on the heat transfer due to low effectiveness values and increased heat transfer coefficients. At a blowing ratio of $M=0.75$, the cylindrical configuration shows just as good performance data as the complex fanshaped geometry. At higher blowing ratios, the fanshaped hole configuration shows much better overall cooling performance in the near hole region. Here, the film cooling effectiveness decreases significantly for the cylindrical holes due to coolant jet separation, whereas the fanshaped holes provide a very surface protection. Further downstream from $s / D=30$ to $s / D=50$, the NHFR data again show similar performance of the two hole types.

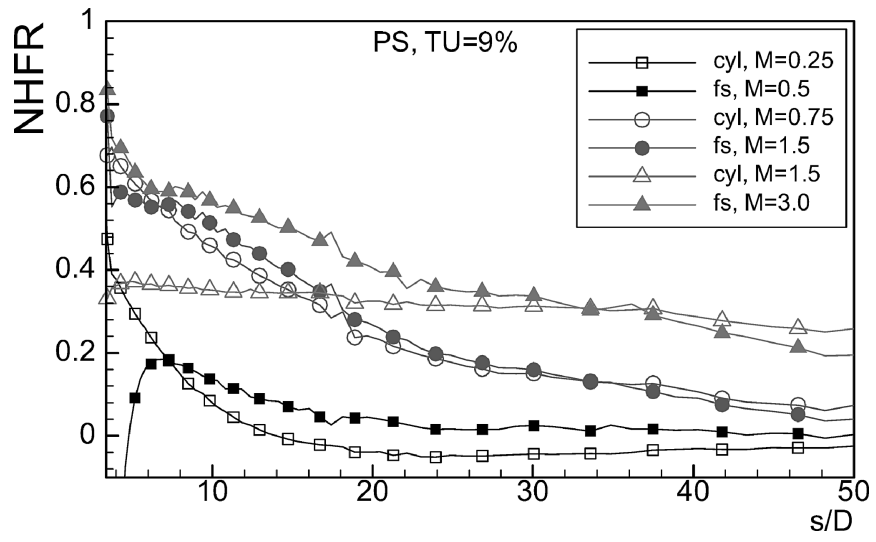

FIGURE 16

Lateral averaged net heat flux reduction parameter for both injection configurations. 


\section{CONCLUSIONS}

Adiabatic film cooling effectiveness and heat transfer measurements have been conducted using a scaled model of a typical guide vane pressure side. The key findings of the experiments can be summarized as follows:

A double row of cylindrical holes provides nearly similar adiabatic film cooling effectiveness values compared to a single row of fanshaped holes only at small blowing ratios. At moderate and high blowing ratios, the fanshaped holes show clearly superior cooling effectiveness. Enhanced mainstream turbulence intensity reduces film cooling effectiveness due to higher diffusion and leads in general to a faster decay of effectiveness in streamwise direction. For cylindrical holes at high blowing ratios, the turbulence intensity has only little effect on adiabatic effectiveness because of jet separation on the one hand and enhanced coolant diffusion on the other hand. The heat transfer coefficient with film cooling is enhanced up to $20 \%$ compared to the case without injection. The main areas with enhanced heat transfer are identified to be located between the injection holes at the lateral edges of the coolant jets. Comparing the overall film cooling performance and taking effectiveness and heat transfer enhancement into account, the two different injection configuration show similar performance until medium blowing ratios. At higher blowing ratios, the fanshaped holes show in total much better surface protection in a region up to a streamwise distance of $s / D=30$.

\section{NOMENCLATURE}

$D \quad$ injection hole diameter

$h \quad$ heat transfer coefficient

$k \quad$ acceleration parameter, $k=\frac{\partial u}{\partial s} \cdot \frac{v}{u^{2}}$

$L \quad$ total chord length of the model

$M \quad$ blowing ratio, $M=\rho_{c} u_{c} / \rho_{m} u_{m}$

NHFR net heat flux reduction parameter, Equation (13)

$\operatorname{Re} \quad$ Reynolds number, $\operatorname{Re}=\frac{u(s) \cdot L}{v}$

$s \quad$ streamwise coordinate

$T U$ mainstream turbulence intensity,

$$
T U=\left(\sqrt{1 / 3 \cdot\left(u_{\mathrm{RMS}}^{2}+v_{\mathrm{RMS}}^{2}+w_{\mathrm{RMS}}^{2}\right)}\right) / u_{\text {mean }}
$$

$u, v, w$ streamwise, normal, and lateral velocity component

$\varepsilon \quad$ emissivity of the painted test plate

$\eta \quad$ adiabatic film cooling effectiveness, Equation (6)

$v \quad$ kinematic viscosity

$\theta \quad$ dimensionless wall temperature, Equation (8)

\section{Subscripts}

$f \quad$ with film injection

$0 \quad$ without film injection

$m \quad$ mainstream gas

c coolant gas

$w \quad$ wall

aw adiabatic wall

sur surrounding

TC thermocouple

RMS root mean square value of velocity fluctuations
Lat lateral averaged

spa space averaged

\section{REFERENCES}

Choe, H., Kays, W. M., and Moffat, R. J. 1974. The superposition approach to film cooling. ASME Paper 74-Wa/GT-27.

Dittmar, J., Jung, I. S., Schulz, A., Wittig, S., and Lee, J. S. 2000. Film cooling from rows of holes-effect of cooling hole shape and row arrangement on adiabatic affectiveness. Heat Transfer in Gas Turbine Systems, Annals of the New York Academy of Science 934:321-328.

Goldstein, R. G. 1971. Film cooling. Advances in Heat Transfer 7:312379.

Goldstein, R. J., Eckert, E. R. G., and Burggraf, F. 1974. Effects of hole geometry and density on three-dimensional film cooling. International Journal of Heat and Mass Transfer 17:595-607.

Gritsch, M., Schulz, A., and Wittig, S. 1997. Adiabatic wall effectiveness measurements of film cooling holes with expanded exits. ASME Paper 97-GT-164.

Gritsch, M., Schulz, A., and Wittig, S. 1998. Heat transfer coefficient measurements of film cooling holes with expanded exits. ASMEPaper 98-GT-28.

Gritsch, M., Baldauf, S., Martiny, M., Schulz, A., and Wittig, S. 1999. The superposition approach to local heat transfer coefficients in high density ratio film cooling flows. ASME Paper 99-GT-168.

Jabbari, M. Y., and Goldstein, R. J. 1978. Adiabatic wall temperature and heat transfer downstream of injection through two rows of holes. Journal of Engineering for Power 100:303-307.

Jubran, B., and Brown, A. 1985. Film cooling from two rows of holes inclined in the streamwise and spanwise directions. Journal of Engineering for Gas Turbine and Power 107:84-91.

Jubran, B. A., and Maiteh, B. Y. 1999. Film cooling and heat transfer from a combination of two rows of simple and/or compound angle holes in inline and/or staggered configurations. Heat and Mass Transfer 34:495-502.

Ligrani, P. M., Wigle, J. M., Ciriello, S., and Jackson, S. M. 1994. Film-cooling from holes with compound angle orientations: Part1Results downstream of two staggered rows of holes with 3D spanwise spacing. Journal of Heat and Mass Transfer 116:341-352.

Makki, Y., and Jakubowski, G. 1986. An experimental study of film cooling from diffused trapezoidal shaped holes. AIAA-Paper 861326.

Metzger, D. E., Carper, H. J., and Swank, L. R. 1968. Heat transfer with film cooling near non-tangential injection slots. ASME Journal of Engineering for Power 90:157-163.

Press, W. H., Flannery, B. P., Teukolsky, S. A., and Vetterling, W. T. 1988. Numerical Recipes, Cambridge University Press, Cambridge, UK.

Reiss, H., and Bölcs, A. 1999. Experimental study of showerhead cooling on a cylinder comparing several configurations using cylindrical and shaped holes. ASME Paper 99-GT-123.

Sen, B., Schmidt, D. L., and Bogard, D. G. 1996. Film cooling with compound angle holes: heat transfer. ASME Journal of Turbomachinery 118:800-806.

Thole, K., Gritsch, M., Schulz, A., and Wittig, S. 1996. Flow field measurements for film cooling holes with expanded exits. ASMEPaper 96-GT-174.

Yu, Y., Yen, C.-H., Shih, T. I.-P., Chyu, M. K., and Gogineni, S. 1999. Film cooling effectiveness and heat transfer coefficient distribution around diffusion shaped holes. ASME Paper 99-GT-34. 

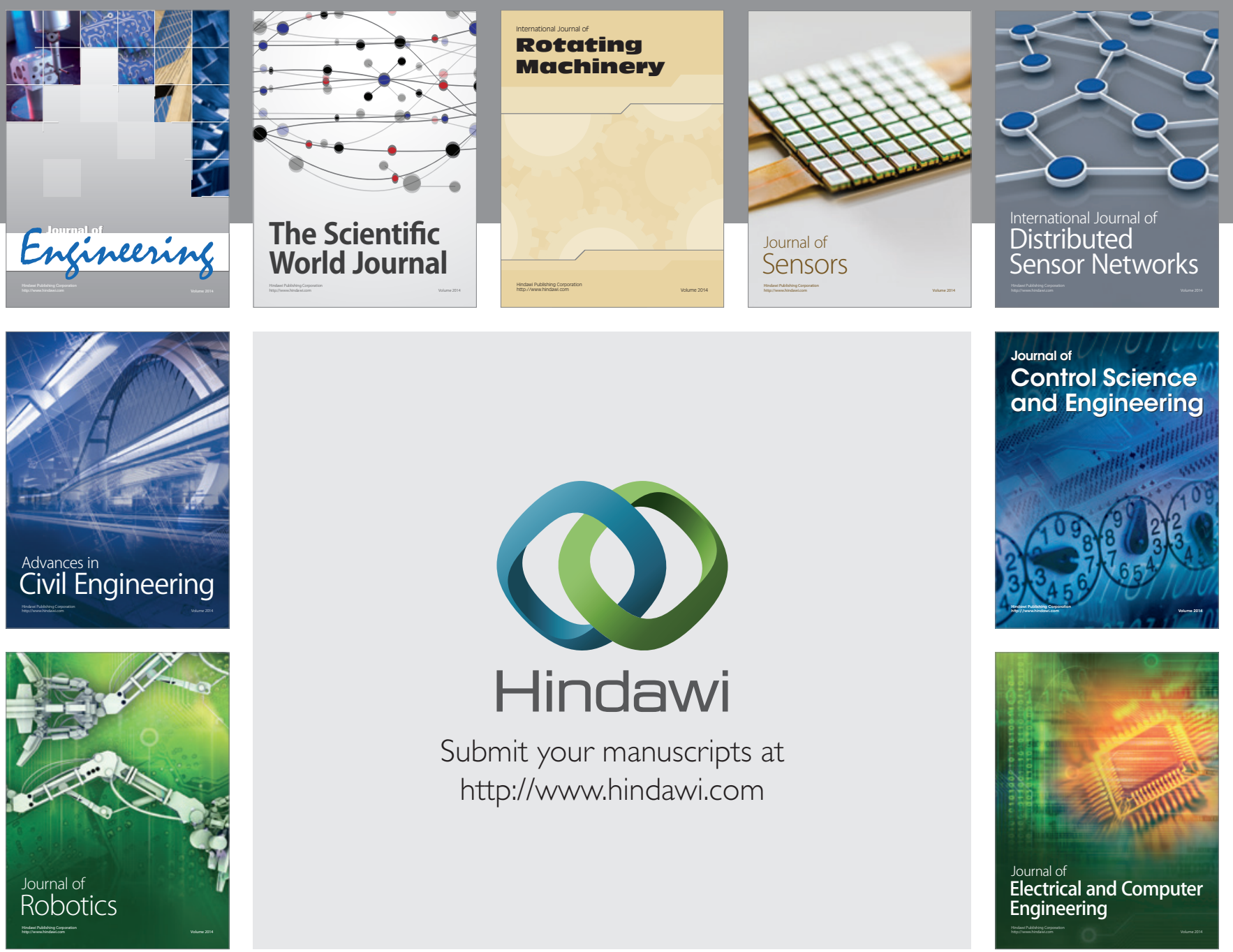

Submit your manuscripts at

http://www.hindawi.com
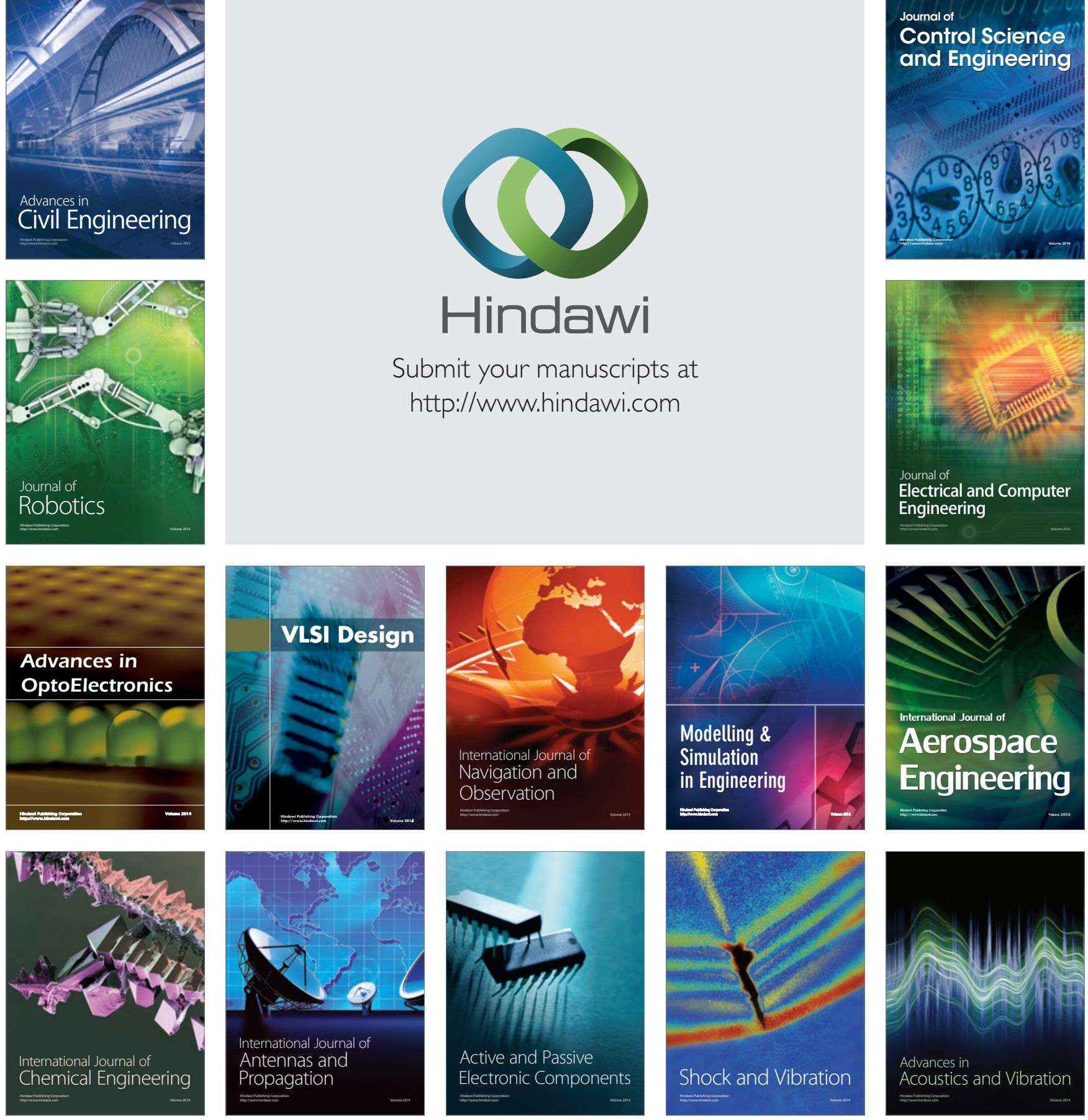